\title{
Anti-VEGF treatment of macular edema associated with retinal vein occlusion: patterns of use and effectiveness in clinical practice (ECHO study report 2)
}

This article was published in the following Dove Press journal: Clinical Ophthalmology

J Michael Jumper'

Pravin U Dugel ${ }^{2,3}$

Sanford Chen ${ }^{4}$

Kevin J Blinder ${ }^{5}$

John G Walt ${ }^{6}$

On behalf of the ECHO Study Group

'West Coast Retina Medical Group, San Francisco, CA, USA; ${ }^{2}$ Retinal Consultants of Arizona, Phoenix, AZ, USA; ${ }^{3}$ USC Roski Eye Institute, Keck School of Medicine, University of Southern California, Los Angeles, CA, USA; ${ }^{4}$ Orange County Retina, Santa Ana, CA, USA; ${ }^{5}$ The Retina Institute, St Louis, MO, USA; ${ }^{6}$ Allergan plc, Irvine, CA, USA
Correspondence: J Michael Jumper West Coast Retina Medical Group, I 445 Bush Street, San Francisco, CA 94109 , USA

Tel +I 4159724600

Fax + I 4159750999

Email jmichaeljumper@gmail.com
Purpose: To evaluate the efficacy, safety, and injection frequency of vascular endothelial growth factor (VEGF) antagonists in the treatment of macular edema secondary to retinal vein occlusion (RVO) in clinical practice.

Patients and methods: A multicenter retrospective study of the medical records of 165 patients (95 branch RVO, 70 central RVO) treated with at least three anti-VEGF injections in the study eye was conducted. Available data collected for at least 6 months after the first injection included Snellen best-corrected visual acuity (BCVA), central retinal thickness (CRT) by time-domain optical coherence tomography (TD-OCT) or spectral-domain optical coherence tomography (SD-OCT), anti-VEGF injections, other treatments/procedures for RVO, and adverse events.

Results: At baseline prior to anti-VEGF treatment, mean BCVA was 20/80 Snellen equivalent and mean CRT was $499 \mu \mathrm{m}$. Mean number of anti-VEGF injections received was 7.1 during the first year, 5.4 during the second year, and 5.9 during the third year; $51.3 \%$ $(842 / 1,641)$ of injections were ranibizumab, $44.1 \%(724 / 1,641)$ were bevacizumab, and $4.6 \%$ $(75 / 1,641)$ were aflibercept. One in five patients received concomitant focal laser treatment. The percentage of patients achieving both BCVA of 20/40 or better and CRT $\leq 250 \mu \mathrm{m}$ on TD-OCT or $\leq 300 \mu \mathrm{m}$ on SD-OCT at the same visit (primary endpoint) was $26.1 \%(30 / 115)$ after the first anti-VEGF injection and ranged from $20.0 \%(7 / 35)$ to $36.7 \%(11 / 30)$ after the first 16 injections. After each anti-VEGF injection from the 1 st to the 16 th, $<60 \%$ of patients achieved $20 / 40$ or better BCVA and $\leq 70 \%$ of patients achieved CRT $\leq 250 \mu \mathrm{m}$ on TD-OCT or $\leq 300 \mu \mathrm{m}$ on SD-OCT. The most common treatment-related adverse event was blurry or cloudy vision.

Conclusion: In this real-world study, a mean of five to seven anti-VEGF injections was administered yearly, and the response to anti-VEGF therapy was suboptimal in many patients. Anti-VEGF therapy was well tolerated.

Keywords: aflibercept, bevacizumab, branch retinal vein occlusion, central retinal vein occlusion, ranibizumab, visual acuity

\section{Introduction}

Retinal vein occlusion (RVO) is a prevalent vision-threatening disease estimated to affect 16.4 million adults worldwide. ${ }^{1}$ RVOs are classified based on the site of the occlusion as branch retinal vein occlusion (BRVO), central retinal vein occlusion (CRVO), and hemiretinal vein occlusion. Macular edema is a common complication 
and a primary cause of vision loss in all forms of RVO. ${ }^{2-4}$ Early treatment of RVO-associated macular edema is associated with better long-term visual outcomes. ${ }^{5-8}$

Standard care for RVO-associated macular edema is intravitreal treatment with a vascular endothelial growth factor (VEGF) inhibitor, most commonly ranibizumab (Lucentis; Genentech, South San Francisco, CA, USA), bevacizumab (Avastin; Genentech, South San Francisco, CA, USA), or aflibercept (Eylea; Regeneron Pharmaceuticals, Tarrytown, NY, USA). Ranibizumab is a humanized antigen-binding fragment of a mouse monoclonal antibody to VEGF with several selective mutations to increase its binding affinity; ranibizumab binds to and inhibits all biologically active forms of VEGF A. ${ }^{9}$ Bevacizumab is a humanized fulllength antibody derived from the same mouse monoclonal antibody, and it also binds to and inhibits all biologically active forms of VEGF A. ${ }^{9}$ Aflibercept is a recombinant fusion protein containing VEGF-binding domains of human VEGF receptors 1 and 2, fused to the Fc portion of the human IgG1 immunoglobulin. It binds to and inhibits all VEGF A isoforms, as well as VEGF B and placenta-derived growth factor. ${ }^{10}$ Ranibizumab and aflibercept are approved by the US Food and Drug Administration for treatment of macular edema following RVO, and bevacizumab is used off-label for this indication.

In the controlled clinical trials conducted for regulatory approval of ranibizumab for treatment of BRVO- and CRVOassociated macular edema (BRAVO ${ }^{5,11}$ and $\mathrm{CRUISE}^{12}$ ), ranibizumab was administered monthly for 6 months, followed by as-needed administration through 1 year. In the BRAVO study, patients with BRVO treated with ranibizumab $0.5 \mathrm{mg}$ had gained a mean of 18.3 letters in best-corrected visual acuity (BCVA) and 64.9\% had achieved 20/40 or better Snellen equivalent BCVA at month $6 .{ }^{11}$ On average, the BCVA gains were sustained at month 12 after a mean of 2.8 additional injections. ${ }^{5}$ The percentage of patients achieving normal central retinal thickness (CRT) of $\leq 250 \mu \mathrm{m}$ was $84.7 \%$ at 6 months and $86.3 \%$ at 12 months. ${ }^{5}$ In the CRUISE study, patients with CRVO received a mean of 3.6 as-needed ranibizumab $0.5 \mathrm{mg}$ injections after the initial six monthly injections. ${ }^{12}$ The mean BCVA gain from baseline in these patients was 14.9 letters at 6 months and 13.9 letters at 12 months, with achievement of $20 / 40$ or better BCVA by $46.9 \%$ of patients at 6 months and $43.1 \%$ of patients at 12 months. ${ }^{12}$ CRT of $\leq 250 \mu \mathrm{m}$ was achieved by $76.9 \%$ of patients at 6 months and $77.7 \%$ of patients at 12 months. ${ }^{12}$ In the VIBRANT registration study of aflibercept for treatment of BRVO-associated macular edema, aflibercept was administered every 4 weeks through week 24 , then every
8 weeks through week $48 .{ }^{13}$ The mean gain in BCVA from baseline was 17.0 letters and the mean improvement in CRT was $281 \mu \mathrm{m}$ at week 24, and these improvements were sustained on average through week $52 .{ }^{13}$ The COPERNICUS and GALILEO studies evaluated aflibercept administered every 4 weeks through week 20 (six injections), then asneeded through week 52 in patients with CRVO-associated macular edema. ${ }^{14,15}$ The mean number of aflibercept injections administered during the year was 11.8 in GALILEO $^{15}$ and not reported for COPERNICUS. Mean BCVA gains from baseline were 17.7 and 18.0 letters at week 24 and 16.2 and 16.9 letters at week 52 in COPERNICUS and GALILEO, respectively, whereas mean CRT changes from baseline were -457 and $-449 \mu \mathrm{m}$ at week 24 and -413 and $-424 \mu \mathrm{m}$ at week 52 in COPERNICUS and GALILEO, respectively. ${ }^{14,15}$ Both ranibizumab and aflibercept demonstrated favorable safety and tolerability profiles in the registration studies. ${ }^{5,12-15}$

There is evidence that anti-VEGF injections are administered less frequently in clinical practice than in the large registration studies. ${ }^{16,17}$ In a study using health insurance claims from a database covering 64 million individuals in the USA from 2008 to 2011, almost all anti-VEGF injections administered for treatment of RVO were bevacizumab, and for patients who began treatment with bevacizumab in 2010, the mean annual number of injections was only 3.3 for patients with BRVO and 3.5 for patients with CRVO. ${ }^{17}$ Frequent intravitreal injections can be burdensome for patients, but the effectiveness of anti-VEGF therapy may be reduced if patients do not receive the recommended monthly loading doses of anti-VEGF therapy and additional as-needed injections. As there is a need to understand the effectiveness of anti-VEGF therapy as used in patients with RVO in the clinical practice setting, the purpose of this study was to evaluate the efficacy, safety, and injection frequency of antiVEGF therapy (ranibizumab, bevacizumab, or aflibercept) as used in clinical practice for the treatment of RVO-associated macular edema.

\section{Patients and methods}

The ECHO study was a multicenter (10 sites), retrospective, open-label chart review study of anti-VEGF use and effectiveness in patients who received at least three intravitreal injections of anti-VEGF for treatment of macular edema secondary to RVO or diabetic macular edema (DME). The study protocol called for subgroup analysis of the results based on the patient diagnosis (RVO-associated macular edema or DME). Results in the patients with DME were reported previously. ${ }^{18}$ 
The study adhered to the tenets of the Declaration of Helsinki and was approved for each site by a central institutional review board, Sterling Institutional Review Board (Atlanta, GA, USA). All patients provided written informed consent. The study was registered with the identifier NCT01918371 at www.ClinicalTrials.gov.

A review of patient records was conducted between August 2013 and September 2014. Patients selected for the study were at least 18 years of age, had RVO-associated macular edema in the study eye that had been treated with at least three anti-VEGF intravitreal injections during the period from June 2010 to the time of the chart review, and had data available for a minimum of 6 months after the first anti-VEGF injection. Patients who received intravitreal antiVEGF treatment for RVO-associated macular edema before June 2010, or who received the anti-VEGF injections as part of or during a clinical study, were excluded. When both eyes of a patient were eligible for the study, the eye that had been treated with the higher number of anti-VEGF injections was selected to be the study eye.

Demographic data and medical and ophthalmic histories were collected from patient records of the baseline visit, when the first anti-VEGF injection was administered. Efficacy and safety data collected for that visit and all ensuing visits for at least the next 6 months included Snellen BCVA, CRT in the $1 \mathrm{~mm}$ central subfield on time-domain optical coherence tomography (TD-OCT) or spectral-domain optical coherence tomography (SD-OCT), anti-VEGF injections, other treatments or procedures for RVO-associated macular edema, adverse events (AEs), biomicroscopy/ophthalmoscopy findings, intraocular pressure (IOP), and cataract and glaucoma surgeries.

The study endpoints were described in detail previously. ${ }^{18}$ The primary endpoint was a combined endpoint of visual and anatomic outcomes, that is, the percentage of patients with both BCVA of $20 / 40$ or better and CRT $\leq 250 \mu \mathrm{m}$ on TD-OCT or $\leq 300 \mu \mathrm{m}$ on SD-OCT at the same visit. Key secondary endpoints included mean number of anti-VEGF injections in each study year, mean time between antiVEGF injections, mean change in BCVA from baseline, mean change in BCVA from baseline across all anti-VEGF injections, mean change in CRT from baseline, percentage of patients achieving BCVA of 20/40 or better, and percentage of patients achieving CRT $\leq 250 \mu \mathrm{m}$ on TD-OCT or $\leq 300 \mu \mathrm{m}$ on SD-OCT.

The statistical analysis of the data was performed using SAS version 9.4 software (SAS Institute Inc., Cary, NC, USA) and a two-sided alpha level of 0.05 . All analyses were based on observed values with no imputation of missing values.
Patients had to have both BCVA and CRT data from the same visit in order to be included in the analysis of the primary endpoint. Snellen BCVA values were converted to approximate Early Treatment Diabetic Retinopathy Study (ETDRS) line scores for analysis, with one ETDRS line being equivalent to five ETDRS letters, using the method of Gregori et al. ${ }^{19}$ Analysis of efficacy data (BCVA and CRT) by anti-VEGF injection number used the BCVA and CRT values representing the greatest improvement from baseline after the injection and before the next injection (peak effect). Change in BCVA from baseline across all anti-VEGF injections was determined using the maximal improvement in BCVA after each injection and an area-under-the-curve approach.

The figures present efficacy results for the first 16 injections because BCVA and CRT data were available for fewer than 20 patients after subsequent injections. Paired $t$-tests were used to evaluate changes in BCVA and CRT from baseline.

\section{Results}

\section{Patient population}

The study included 165 patients with RVO (57.6\% with BRVO and $42.4 \%$ with CRVO) and related macular edema that was treated with at least three injections of anti-VEGF therapy. Baseline characteristics of the patients are listed in Table 1. Most patients were white, and the mean age was 69.4 years. The median duration of RVO in the study eye at baseline was 0 weeks, suggesting that the majority of patients received their first anti-VEGF injection within a week of RVO diagnosis.

A total of $117(70.9 \%)$ patients had at least 1 year of follow-up data available after initiation of anti-VEGF treatment. Among these patients, 40 (24.2\%) continued to receive anti-VEGF therapy and had follow-up data available through year 2, and 17 (10.3\%) continued to receive anti-VEGF therapy and had follow-up data available through year 3 .

\section{Treatment patterns}

Of the 1,641 anti-VEGF injections administered in the study eyes during the study period, $842(51.3 \%)$ were ranibizumab, 724 (41.1\%) were bevacizumab, and 75 (4.6\%) were aflibercept. Most patients received repeated injections of a single anti-VEGF agent, but in $33.3 \%$ (55/165) of patients, the type of anti-VEGF therapy used was switched at least once during the study period. The most common switch in anti-VEGF therapy was from ranibizumab to bevacizumab $(24.2 \%$ of patients, 40/165).

The number of anti-VEGF injections received by patients in each study year was analyzed for patients who received at 
Table I Baseline patient demographics

\begin{tabular}{|c|c|}
\hline Parameters & $\begin{array}{l}\text { Patient } \\
\text { population } \\
(\mathrm{N}=165)\end{array}$ \\
\hline Age, mean (SD), years & $69.4(13.6)$ \\
\hline \multicolumn{2}{|l|}{ Race, $n$ (\%) } \\
\hline White & $114(69.1)$ \\
\hline Asian & $13(7.9)$ \\
\hline Black or African-American & $6(3.6)$ \\
\hline Other or not recorded & $32(19.4)$ \\
\hline \multicolumn{2}{|l|}{ RVO diagnosis in the study eye, $\mathrm{n}(\%)$} \\
\hline BRVO & $95(57.6)$ \\
\hline CRVO & $70(42.4)$ \\
\hline \multicolumn{2}{|l|}{ Ischemic RVO, n (\%) } \\
\hline Yes & $14(8.5)$ \\
\hline No & $60(36.4)$ \\
\hline Not recorded & $91(55.1)$ \\
\hline Median duration of RVO in the study eye, weeks & 0 \\
\hline \multicolumn{2}{|l|}{ History of procedures in the study eye, $n(\%)$} \\
\hline Retinal focal laser & $10(6.1)$ \\
\hline Pars plana vitrectomy & $5(3.0)$ \\
\hline Panretinal photocoagulation & $4(2.4)$ \\
\hline Trabeculectomy & $4(2.4)$ \\
\hline Intravitreal injection of dexamethasone implant & $4(2.4)$ \\
\hline Intravitreal injection of triamcinolone acetonide & $3(1.8)$ \\
\hline \multicolumn{2}{|l|}{ Lens status in the study eye, $\mathrm{n}(\%)$} \\
\hline Phakic & $117(70.9)$ \\
\hline Pseudophakic & $38(23.0)$ \\
\hline Not recorded & $10(6.1)$ \\
\hline \multicolumn{2}{|l|}{ Current concomitant diagnosis in the study eye, $\mathrm{n}(\%)$} \\
\hline Glaucoma & $28(17.0)$ \\
\hline Ocular hypertension & $5(3.0)$ \\
\hline Using IOP-lowering medication in the study eye, $n(\%)$ & $28(17.0)$ \\
\hline BCVA in the study eye, mean (SD), ETDRS lines ${ }^{a}$ & $10.6(4.0)$ \\
\hline Snellen equivalent & $20 / 80$ \\
\hline CRT in the study eye, mean (SD), $\mu \mathrm{m}$ & $499(188)$ \\
\hline
\end{tabular}

Note: ${ }^{a}$ One ETDRS line $=$ five ETDRS letters.

Abbreviations: BCVA, best-corrected visual acuity; BRVO, branch retinal vein occlusion; CRT, central retinal thickness; CRVO, central retinal vein occlusion; ETDRS, Early Treatment Diabetic Retinopathy Study; IOP, intraocular pressure; RVO, retinal vein occlusion.

least one anti-VEGF injection during the year and had data available throughout the year (Figure 1). Patients received a mean of 7.1 (range, 1-15) anti-VEGF injections during year 1, 5.4 (range, 1-13) anti-VEGF injections during year 2, and 5.9 (range, 1-11) anti-VEGF injections during year 3. The mean interval between anti-VEGF injections ranged from 1.1 to 1.6 months across the first 16 anti-VEGF injections (Figure 2).

In addition to the 1,641 intravitreal injections of antiVEGF antagonists, 100 intravitreal injections of corticosteroid (98 injections of dexamethasone intravitreal implant and 2 injections of triamcinolone acetonide) were administered in the study eyes during the study period. Furthermore, one in five study eyes received adjunctive treatment with focal laser photocoagulation during the study period (Table 2).

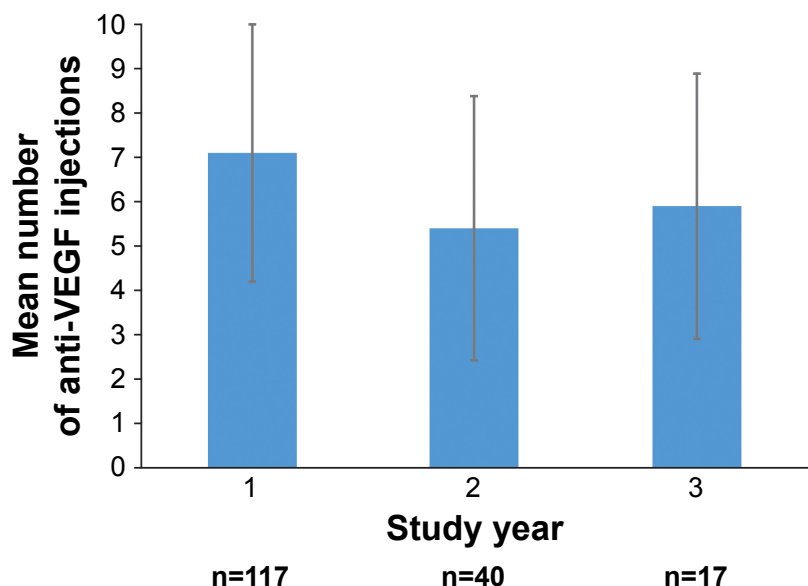

Figure I Number of anti-VEGF injections received by patients each study year. Notes: Patients included in the analysis for a particular year received at least one anti-VEGF injection during the year and had data available for a minimum of 50 weeks (year I), 100 weeks (year 2), or 150 weeks (year 3). Error bars indicate the SD.

Abbreviation: VEGF, vascular endothelial growth factor.

\section{Efficacy outcomes}

The primary endpoint of combined visual and anatomic response (BCVA of 20/40 or better and CRT $\leq 250 \mu \mathrm{m}$ on TD-OCT or $\leq 300 \mu \mathrm{m}$ on SD-OCT at the same visit) was achieved by $20.0 \%-36.7 \%$ of patients after the first 16 antiVEGF injections (Figure 3). The percentage of patients who achieved 20/40 or better BCVA ranged from 40.0\% $(10 / 25)$ to $56.7 \%(76 / 134)$ after these injections (Figure 4), and the percentage of patients who achieved CRT $\leq 250 \mu \mathrm{m}$ on TD-OCT or $\leq 300 \mu \mathrm{m}$ on SD-OCT ranged from $50.4 \%$ $(67 / 133)$ to $69.6 \%(32 / 46)$, as shown in Figure 5.

Mean changes in BCVA from baseline after anti-VEGF injections are shown in Figure 6. The mean (SD) change from baseline BCVA after the first 16 anti-VEGF injections

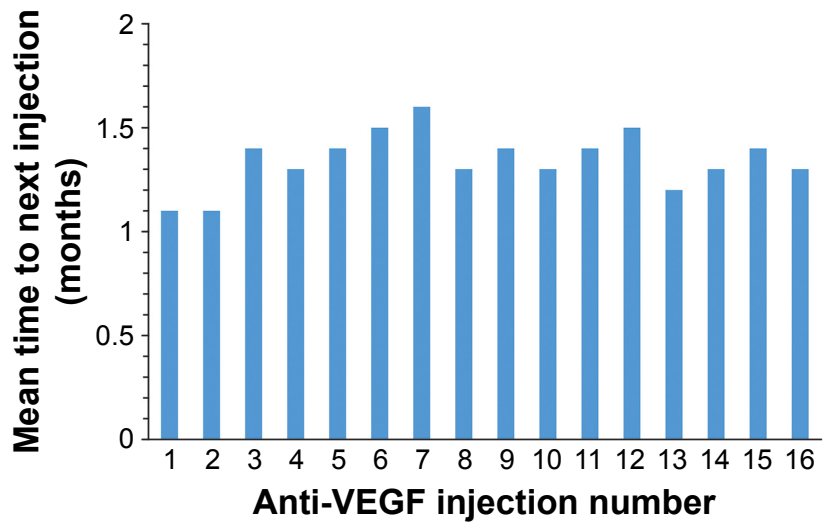

Figure 2 Anti-VEGF injection interval.

Notes: Mean time to next injection was analyzed for all patients who received another anti-VEGF injection within the study period. The number of patients with data for injections I through 16 was 163, 162, I57, 143, I27, II4, 99, 82, 73, 63, 50, $46,38,34,31$, and 27, respectively.

Abbreviation: VEGF, vascular endothelial growth factor. 
Table 2 Procedures in study eyes during the study period

\begin{tabular}{ll}
\hline Procedures, $\mathbf{n}(\%)$ & $\begin{array}{l}\text { Patients } \\
\mathbf{( N = 1 6 5 )}\end{array}$ \\
\hline Laser photocoagulation & $33(20.0)$ \\
$\quad$ Focal laser & $9(5.5)$ \\
$\quad$ Panretinal photocoagulation & \\
Glaucoma surgeries & $\mathrm{I}(0.6)$ \\
$\quad$ Laser & $2(1.2)$ \\
$\quad$ Incisional & $20(17.1)^{\mathrm{a}}$ \\
Cataract surgery in baseline phakic eyes $(\mathrm{n}=117)$ &
\end{tabular}

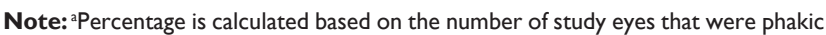
at baseline.

ranged from $+1.4(4.2)$ to +2.3 (4.0) approximate ETDRS lines. The mean $\pm \mathrm{SD}$ change in BCVA from baseline across all injections administered during the study period was $+2.0 \pm 3.9$ approximate ETDRS lines $(P<0.001)$.

The CRT value in the $1 \mathrm{~mm}$ central subfield, evaluated on either TD-OCT or SD-OCT, was used for the calculation of mean CRT. The mean CRT in the study eyes decreased significantly after anti-VEGF treatment, but remained elevated (Figure 7). The mean change in CRT from baseline ranged from -152 to $-255 \mu \mathrm{m}$ after the first 16 anti-VEGF injections, and the mean CRT ranged from 309 to $361 \mu \mathrm{m}$ after these injections (Figure 7).

\section{Safety outcomes}

Sixty-six treatment-related AEs were reported during the study period. All of the treatment-related AEs were ocular; the most common were blurry or cloudy vision $(n=11)$, focal

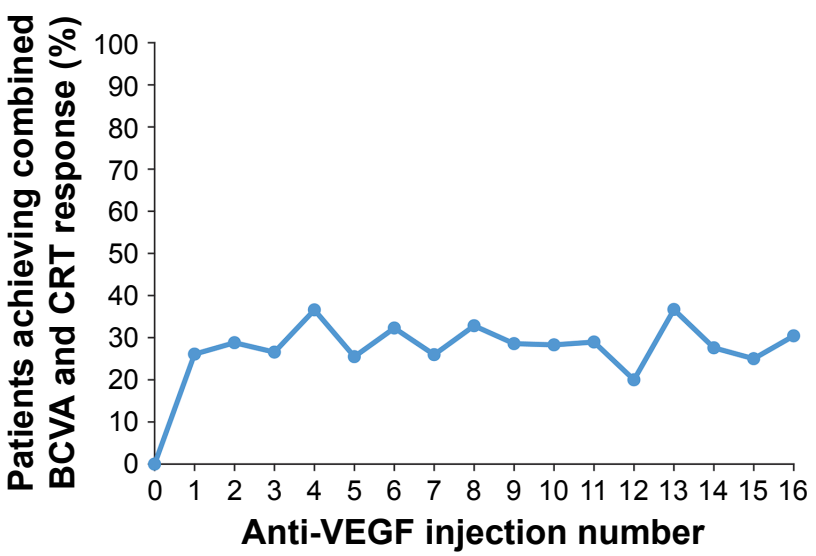

Figure 3 Percentage of patients who met the primary endpoint after each antiVEGF injection.

Notes: The primary endpoint was a combined endpoint of both BCVA response (defined as 20/40 or better BCVA) and CRT response (defined as CRT $\leq 250 \mu \mathrm{m}$ on TD-OCT or $\leq 300 \mu \mathrm{m}$ on SD-OCT) at the same visit. Patients had to have both BCVA and CRT data from the same visit to be included in the analysis. The number of patients with data for injections 0 through 16 was 105, 115, 118, 128, 123, 106, $96,77,64,63,53,38,35,30,29,24$, and 23 , respectively.

Abbreviations: BCVA, best-corrected visual acuity; CRT, central retinal thickness; SD-OCT, spectral-domain optical coherence tomography; TD-OCT, time-domain optical coherence tomography; VEGF, vascular endothelial growth factor.

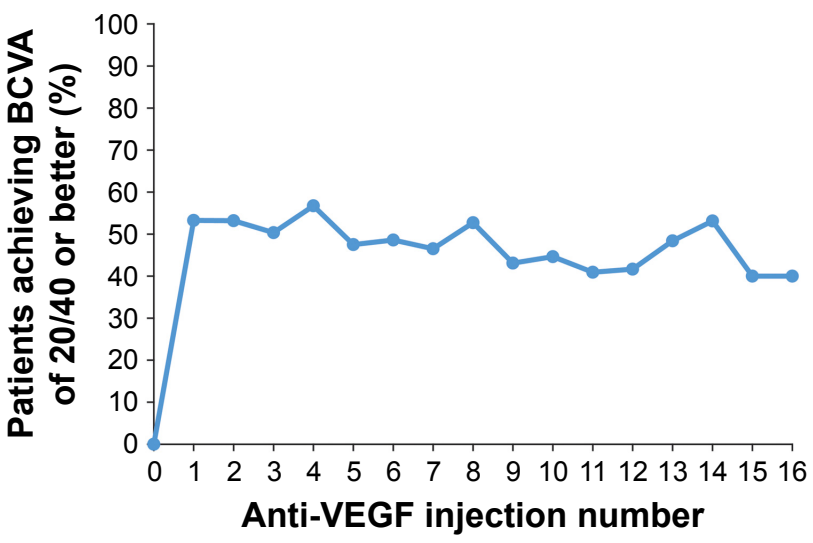

Figure 4 Percentage of patients with 20/40 or better BCVA after each anti-VEGF injection.

Note: The number of patients with BCVA data after injections 0 through 16 was $133,139,141,14 \mid, 134,120,107,86,74,65,56,44,36,31,32,25$, and 25, respectively.

Abbreviations: BCVA, best-corrected visual acuity; VEGF, vascular endothelial growth factor.

or panretinal photocoagulation scars $(\mathrm{n}=9)$, subconjunctival hemorrhage $(n=7)$, elevated IOP or glaucoma $(n=6)$, eye redness $(\mathrm{n}=6)$, and floaters $(\mathrm{n}=5)$. Of the 12 serious AEs reported, only one (spots of blurred vision in the treated eye) was considered by the investigator to be related to treatment.

An increase in IOP of $>10 \mathrm{mmHg}$ from baseline was reported in the study eye of $16(9.7 \%)$ patients. Eighteen $(10.9 \%)$ study eyes had an IOP $>25 \mathrm{mmHg}$ during the study period, and $2(1.2 \%)$ had an IOP $>35 \mathrm{mmHg}$.

\section{Discussion}

In this retrospective study of the medical charts of patients with RVO treated with anti-VEGF therapy at 10 clinical

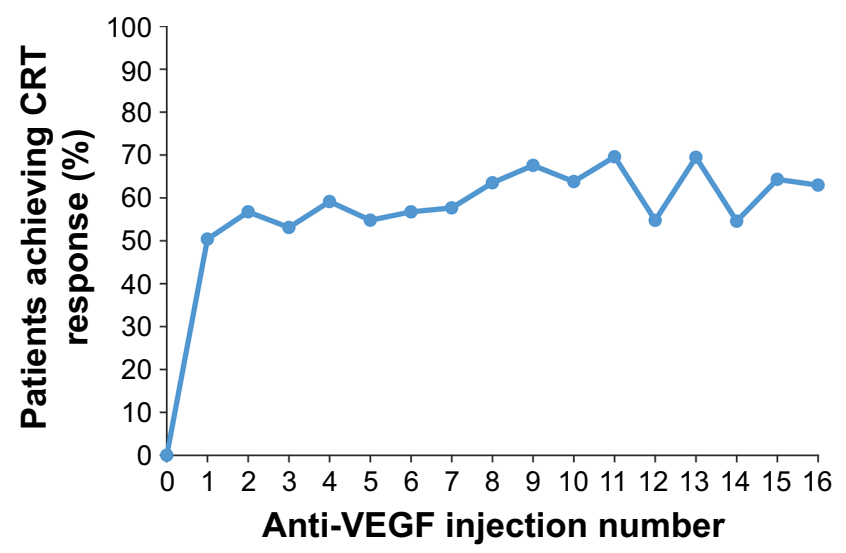

Figure 5 Percentage of patients with CRT $\leq 250 \mu \mathrm{m}$ on TD-OCT or $\leq 300 \mu \mathrm{m}$ on SD-OCT after each anti-VEGF injection.

Note: The number of patients with CRT data after injections 0 through 16 was 128 , 133, 134, 145, 137, II5, 104, 85, 74, 74, 58, 46, 42, 36, 33, 28, and 27, respectively. Abbreviations: CRT, central retinal thickness; SD-OCT, spectral-domain optical coherence tomography; TD-OCT, time-domain optical coherence tomography; VEGF, vascular endothelial growth factor. 


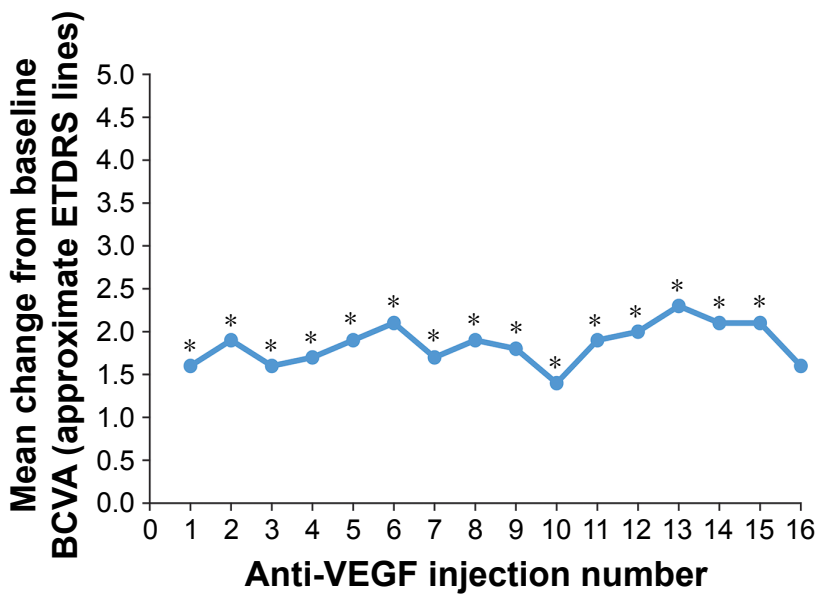

Figure 6 Mean change in BCVA from baseline after each anti-VEGF injection. Notes: The number of patients with change from baseline BCVA data after injections I through 16 was II6, II5, II8, I09, I0I, 89, 73, 66, 58, 49, 36, 34, 25, 27,23 , and 22 , respectively. $* P \leq 0.031$.

Abbreviations: BCVA, best-corrected visual acuity; ETDRS, Early Treatment Diabetic Retinopathy Study; VEGF, vascular endothelial growth factor.

sites, anti-VEGF injections were administered less frequently and were less effective than in the registration randomized controlled studies of ranibizumab and aflibercept for treatment of macular edema related to RVO. Patients received a mean of 7.1, 5.4, and 5.9 anti-VEGF injections during the first, second, and third year of therapy, respectively, and the mean improvement in BCVA after the first 16 injections ranged from 1.4 to 2.3 lines. As the aim of treatment for RVO-associated macular edema is to achieve both good visual acuity and a dry macula, the primary endpoint of the study was a combined endpoint of BCVA of 20/40 or better and CRT $\leq 250 \mu \mathrm{m}$ on TD-OCT or $\leq 300 \mu \mathrm{m}$ on SD-OCT at the same visit. Only $20.0 \%-36.7 \%$ of patients achieved this endpoint after the first 16 anti-VEGF injections.
Most patients in this study were diagnosed with BRVO, consistent with the reported higher prevalence of BRVO relative to CRVO in population studies, ${ }^{1}$ but 70 (42.4\%) patients were diagnosed with CRVO. The demographic and clinical characteristics of the patients were representative of patients with RVO seen at ophthalmic practices in the USA. Glaucoma has been identified as a risk factor for CRVO, ${ }^{20}$ and $28(17.0 \%)$ patients had glaucoma as well as RVO in the study eye. Patients typically received their first anti-VEGF treatment within a week of RVO diagnosis and generally had substantial vision loss and thickening of the retina, with mean BCVA of 20/80 Snellen and mean CRT of $499 \mu \mathrm{m}$, when anti-VEGF treatment was initiated.

The majority of patients were treated with the same antiVEGF agent throughout the study period, but in one-third of the patients, the type of anti-VEGF used was switched at least once, usually from ranibizumab to bevacizumab. The reasons for switching between anti-VEGF therapies were not collected, but as controlled clinical studies ${ }^{21,22}$ and retrospective case series studies ${ }^{23,24}$ have suggested that ranibizumab and bevacizumab are similarly effective in the treatment of patients with RVO, and bevacizumab is available at substantially lower cost,${ }^{25}$ patients may have switched from ranibizumab to bevacizumab for cost savings.

It is acknowledged that monthly visits and intravitreal injections can be burdensome for patients. In this study, the mean number of anti-VEGF injections received by patients in the first, second, and third year was lower than if injections had been administered monthly throughout the year. The mean annual number of anti-VEGF injections could have been reduced in part because some patients stopped treatment due to lack of efficacy or resolution of the macular edema.
A

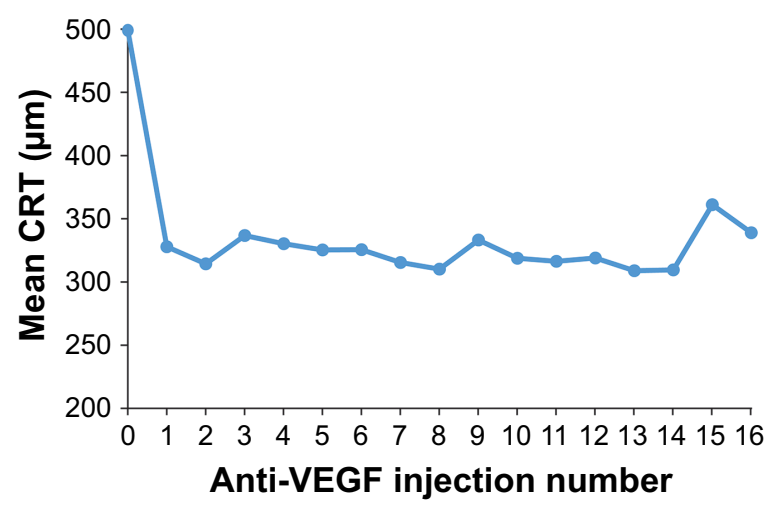

B

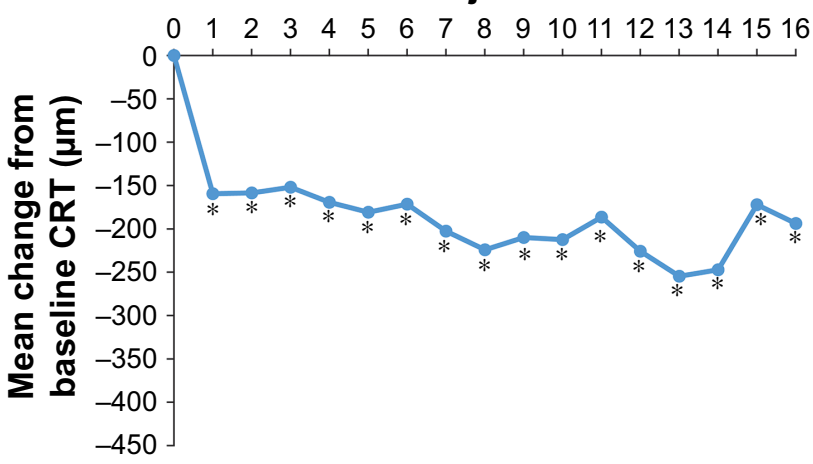

Figure 7 Mean CRT (A) and mean change in CRT from baseline (B) after each anti-VEGF injection.

Notes: The number of patients with CRT data after injections 0 through 16 was I28, 133, 134, I45, I37, II5, 104, 85, 74, 74, 58, 46, 42, 36, 33, 28, and 27, respectively. The number of patients with change from baseline CRT data after injections I through 16 was II0, II0, II , 109, 93, 85, 70, 63, 65, 48, 40, 36, 29, 27, 22, and 21 , respectively. $* P \leq 0.039$.

Abbreviations: CRT, central retinal thickness; VEGF, vascular endothelial growth factor. 
However, the mean interval between anti-VEGF injections was consistently longer than 1 month. After the first and second anti-VEGF injections, the mean time to the next injection was 1.1 month, suggesting that many patients received an initial three loading injections of anti-VEGF at intervals close to 1 month. Longer inter-injection intervals after subsequent anti-VEGF injections could be explained in part by use of treat-and-extend or treat-as-needed treatment protocols. ${ }^{26}$ Missed visits and use of adjunctive therapies between anti-VEGF injections (eg, 20\% of patients received focal laser coagulation during the study) could also have contributed to the reduced frequency of anti-VEGF injections after the third injection. In a recent large retrospective study of patients treated with anti-VEGF using as-needed treatment protocols at one center in Germany, ${ }^{26}$ patients with RVO received a median of six anti-VEGF injections in the first year of treatment and fewer injections in subsequent years, consistent with the results of this study.

Visual and anatomic outcomes were suboptimal in a substantial number of patients in this study. After each of the first 16 injections, over $40 \%$ of patients did not achieve 20/40 or better BCVA and over $30 \%$ of patients did not achieve $\mathrm{CRT} \leq 250 \mu \mathrm{m}$ on TD-OCT or $\leq 300 \mu \mathrm{m}$ on SD-OCT. Some patients who achieved 20/40 or better BCVA failed to meet the primary endpoint because CRT remained $>250 \mu \mathrm{m}$ on TD-OCT or $>300 \mu \mathrm{m}$ on SD-OCT, and conversely, some patients who achieved CRT $\leq 250 \mu \mathrm{m}$ on TD-OCT or $\leq 300 \mu \mathrm{m}$ on SD-OCT failed to meet the primary endpoint because their BCVA remained worse than 20/40. The percentage of patients who achieved CRT $\leq 250 \mu \mathrm{m}$ on TD-OCT or $\leq 300 \mu \mathrm{m}$ on SD-OCT was generally higher than the percentage of patients who achieved 20/40 or better BCVA, possibly because of ischemia, other complications of the RVO, or other ocular conditions that prevented visual acuity gains. The primary endpoint of combined visual and anatomic response was met by only one-third of patients after most injections. We believe that these results are unlikely to be explained solely by inadequate dosing frequency. In the BRAVO and CRUISE registration studies of ranibizumab for treatment of BRVO and CRVO, $\sim 15 \%$ and $23 \%$ of patients, respectively, had residual edema (centerpoint thickness $>250 \mu \mathrm{m}$ on TD-OCT) after six monthly injections of ranibizumab $0.5 \mathrm{mg} .{ }^{27}$ Therefore, some patients can be expected to respond suboptimally to anti-VEGF treatment even with monthly dosing.

VEGF inhibitors are the standard treatment for RVOassociated macular edema because of their safety, as well as their efficacy in reducing macular edema and improving visual acuity in many patients. Anti-VEGF treatment of patients with RVO was demonstrated to be well tolerated in the registration studies of ranibizumab and aflibercept, 5,7,12,13,15 and the results of this study confirmed that anti-VEGF treatment of RVO-associated macular edema is well tolerated in the clinical practice setting. Only one serious AE related to treatment was reported, and there were no reports of thromboembolic events related to anti-VEGF treatment.

This study had the limitations inherent in retrospective chart review studies, including lack of standardization of assessments and missing data. For example, the diagnosis of ischemia was not standardized and the ischemia status of the RVO was unavailable in the majority of patients. Intravitreal corticosteroid injections and focal laser coagulation treatments were received by some patients during the study period and could have affected the observed efficacy outcomes. Also, most of the anti-VEGF injections received by patients were ranibizumab or bevacizumab, because the chart review covered the period from June 2010 to September 2014, and aflibercept was approved for treatment of CRVO-associated macular edema in September 2012 and BRVO-associated macular edema in October 2014. The underrepresentation of aflibercept could have had an effect on efficacy outcomes in this study because there have been reports of patients with CRVO refractory to treatment with ranibizumab or bevacizumab responding favorably to aflibercept. ${ }^{28,29}$ However, a retrospective study of anti-VEGF use in clinical practice reported similar efficacy of aflibercept and ranibizumab in treatment-naive patients with $\mathrm{CRVO},{ }^{30}$ the randomized controlled SCORE2 study demonstrated noninferiority of bevacizumab to aflibercept in improving visual acuity in patients with macular edema secondary to CRVO or hemiretinal vein occlusion, ${ }^{25}$ a network meta-analysis of controlled studies of approved treatments for BRVOassociated macular edema reported no significant differences between ranibizumab and aflibercept, ${ }^{31}$ and a retrospective comparative chart review study reported similar efficacy of bevacizumab and aflibercept in treatment-naive patients with perfused BRVO. ${ }^{32}$ Finally, only patients who received three or more anti-VEGF injections were included in the study. This may have resulted in an underestimation of the efficacy of anti-VEGF treatment, because patients with resolution of macular edema after only one or two anti-VEGF injections, as can occur in BRVO, ${ }^{32}$ would have been excluded.

\section{Conclusion}

This study demonstrated that outcomes of anti-VEGF treatment in the clinical practice setting are suboptimal in a substantial number of patients with RVO when anti-VEGF injections are administered at mean intervals of 1.1-1.6 
months. More frequent injections may improve outcomes, but some patients do not achieve a dry macula even with monthly injections. As long-term, persistent macular edema can lead to irreversible tissue damage and vision loss, other treatment options including intravitreal corticosteroids and laser photocoagulation may be warranted for patients with RVO who respond suboptimally to anti-VEGF therapy.

\section{Acknowledgments}

This study was sponsored by Allergan plc, Dublin, Ireland. Project management, data management, and statistical analysis of the data were performed by Trial Runners, LLC (Dickinson, ND, USA). Writing and editorial assistance was provided to the authors by Kate Ivins, $\mathrm{PhD}$, Evidence Scientific Solution, Philadelphia, PA, USA, and funded by Allergan plc. All authors met the ICMJE authorship criteria. Neither honoraria nor payments were made for authorship. This study was presented, in part, at the Retina Society 48th Annual Meeting, Paris, France, October 9, 2015.

ECHO Study Group Principal Investigators: Kevin J Blinder, MD (St Louis, MO, USA); David S Boyer, MD (Beverly Hills, CA, USA); Antonio Capone Jr, MD (Royal Oak, MI, USA); Sanford Chen, MD (Santa Ana, CA, USA); Pravin Dugel, MD (Phoenix, AZ, USA); J Michael Jumper, MD (San Francisco, CA, USA); Alan Margherio, MD (Grand Rapids, MI, USA); Joel Pearlman, MD (Sacramento, CA, USA); and Michael A Singer, MD (San Antonio, TX, USA).

\section{Disclosure}

JM Jumper is a clinical investigator for Allergan, Genentech, Ophthotech, and Regeneron and has received research grants from Allergan. P Dugel is a consultant to Allergan and has received research grants from Allergan. S Chen is a consultant to Alcon, Alimera, Allergan, Genentech, and Regeneron. KJ Blinder is a consultant to Allergan, Bausch+Lomb, Dutch Ophthalmics, Genentech, and Regeneron and has received lecture fees from Allergan, Bausch+Lomb, and Regeneron. JG Walt is an employee of Allergan. The authors report no other conflicts of interest in this work.

\section{References}

1. Rogers S, McIntosh RL, Cheung N, et al. The prevalence of retinal vein occlusion: pooled data from population studies from the United States, Europe, Asia, and Australia. Ophthalmology. 2010;117(2):313-319.e1.

2. Jaulim A, Ahmed B, Khanam T, Chatziralli IP. Branch retinal vein occlusion: epidemiology, pathogenesis, risk factors, clinical features, diagnosis, and complications. An update of the literature. Retina. 2013;33(5): 901-910.

3. McIntosh RL, Rogers SL, Lim L, et al. Natural history of central retinal vein occlusion: an evidence-based systematic review. Ophthalmology. 2010;117(6):1113-1123.e15.
4. Hayreh SS, Podhajsky PA, Zimmerman MB. Central and hemicentral retinal vein occlusion: role of anti-platelet aggregation agents and anticoagulants. Ophthalmology. 2011;118(8):1603-1611.

5. Brown DM, Campochiaro PA, Bhisitkul RB, et al. Sustained benefits from ranibizumab for macular edema following branch retinal vein occlusion: 12-month outcomes of a phase III study. Ophthalmology. 2011; 118(8):1594-1602.

6. Thach AB, Yau L, Hoang C, Tuomi L. Time to clinically significant visual acuity gains after ranibizumab treatment for retinal vein occlusion: BRAVO and CRUISE trials. Ophthalmology. 2014;121(5): 1059-1066.

7. Brown DM, Heier JS, Clark WL, et al. Intravitreal aflibercept injection for macular edema secondary to central retinal vein occlusion: 1-year results from the phase 3 COPERNICUS study. Am J Ophthalmol. 2013; 155(3):429-437.e7.

8. Yeh WS, Haller JA, Lanzetta P, et al. Effect of the duration of macular edema on clinical outcomes in retinal vein occlusion treated with dexamethasone intravitreal implant. Ophthalmology. 2012;119(6):1190-1198.

9. Steinbrook R. The price of sight-ranibizumab, bevacizumab, and the treatment of macular degeneration. $N$ Engl J Med. 2006;355(14): 1409-1412.

10. Papadopoulos N, Martin J, Ruan Q, et al. Binding and neutralization of vascular endothelial growth factor (VEGF) and related ligands by VEGF Trap, ranibizumab and bevacizumab. Angiogenesis. 2012;15(2): 171-185.

11. Campochiaro PA, Heier JS, Feiner L, et al. Ranibizumab for macular edema following branch retinal vein occlusion: six-month primary end point results of a phase III study. Ophthalmology. 2010;117(6): 1102-1112.e1.

12. Campochiaro PA, Brown DM, Awh CC, et al. Sustained benefits from ranibizumab for macular edema following central retinal vein occlusion: twelve-month outcomes of a phase III study. Ophthalmology. 2011; 118(10):2041-2049.

13. Clark WL, Boyer DS, Heier JS, et al. Intravitreal aflibercept for macular edema following branch retinal vein occlusion: 52-week results of the VIBRANT study. Ophthalmology. 2016;123(2):330-336.

14. Heier JS, Clark WL, Boyer DS, et al. Intravitreal aflibercept injection for macular edema due to central retinal vein occlusion: two-year results from the COPERNICUS study. Ophthalmology. 2014;121(7): 1414-1420.e1.

15. Korobelnik JF, Holz FG, Roider J, et al. Intravitreal aflibercept injection for macular edema resulting from central retinal vein occlusion: one-year results of the phase 3 GALILEO study. Ophthalmology. 2014;121(1): 202-208.

16. Holekamp NM, Liu Y, Yeh WS, et al. Clinical utilization of anti-VEGF agents and disease monitoring in neovascular age-related macular degeneration. Am J Ophthalmol. 2014;157(4):825.e1-833.e1.

17. Kiss S, Liu Y, Brown J, et al. Clinical utilization of anti-vascular endothelial growth-factor agents and patient monitoring in retinal vein occlusion and diabetic macular edema. Clin Ophthalmol. 2014;8: 1611-1621.

18. Blinder KJ, Dugel PU, Chen S, et al. Anti-VEGF treatment of diabetic macular edema in clinical practice: effectiveness and patterns of use (ECHO study report 1). Clin Ophthalmol. 2017;11:393-401.

19. Gregori NZ, Feuer W, Rosenfeld PJ. Novel method for analyzing Snellen visual acuity measurements. Retina. 2010;30(7):1046-1050.

20. Risk factors for central retinal vein occlusion. The Eye Disease CaseControl Study Group. Arch Ophthalmol. 1996;114(5):545-554.

21. Narayanan R, Panchal B, Das T, et al. A randomised, double-masked, controlled study of the efficacy and safety of intravitreal bevacizumab versus ranibizumab in the treatment of macular oedema due to branch retinal vein occlusion: MARVEL report no. 1. Br J Ophthalmol. 2015;99(7):954-959.

22. Rajagopal R, Shah GK, Blinder KJ, et al. Bevacizumab versus ranibizumab in the treatment of macular edema due to retinal vein occlusion: 6-month results of the CRAVE study. Ophthalmic Surg Lasers Imaging Retina. 2015;46(8):844-850. 
23. Yuan A, Ahmad BU, Xu D, et al. Comparison of intravitreal ranibizumab and bevacizumab for the treatment of macular edema secondary to retinal vein occlusion. Int J Ophthalmol. 2014;7(1):86-91.

24. Khan M, Wai KM, Silva FQ, et al. Comparison of ranibizumab and bevacizumab for macular edema secondary to retinal vein occlusions in routine clinical practice. Ophthalmic Surg Lasers Imaging Retina. 2017; 48(6):465-472.

25. Scott IU, VanVeldhuisen PC, Ip MS, et al. Effect of bevacizumab vs. aflibercept on visual acuity among patients with macular edema due to central retinal vein occlusion: the SCORE2 randomized clinical trial. JAMA. 2017;317(20):2072-2087.

26. Wecker T, Ehlken C, Bühler A, et al. Five-year visual acuity outcomes and injection patterns in patients with pro-re-nata treatments for AMD, DME, RVO and myopic CNV. Br J Ophthalmol. 2017;101(3) 353-359.

27. Bhisitkul RB, Campochiaro PA, Shapiro H, Rubio RG. Predictive value in retinal vein occlusions of early versus late or incomplete ranibizumab response defined by optical coherence tomography. Ophthalmology. 2013;120(5):1057-1063.
28. Papakostas TD, Lim L, van Zyl T, et al. Intravitreal aflibercept for macular oedema secondary to central retinal vein occlusion in patients with prior treatment with bevacizumab or ranibizumab. Eye. 2016;30(1):79-84.

29. Konidaris V, Al-Hubeshy Z, Tsaousis KT, Gorgoli K, Banerjee S, Empeslidis T. Outcomes of switching treatment to aflibercept in patients with macular oedema secondary to central retinal vein occlusion refractory to ranibizumab. Int Ophthalmol. Epub 12 Apr 2017.

30. Chatziralli I, Theodossiadis G, Moschos MM, Mitropoulos P Theodossiadis P. Ranibizumab versus aflibercept for macular edema due to central retinal vein occlusion: 18 -month results in real-life data. Graefes Arch Clin Exp Ophthalmol. 2017;255(6):1093-1100.

31. Regnier SA, Larsen M, Bezlyak V, Allen F. Comparative efficacy and safety of approved treatments for macular oedema secondary to branch retinal vein occlusion: a network meta-analysis. BMJ Open. 2015; 5(6): 007527.

32. Wang JK, Su PY, Hsu YR, Chen YJ, Chen FT, Tseng YY. Comparison of the efficacy of intravitreal aflibercept and bevacizumab for macular edema secondary to branch retinal vein occlusion. J Ophthalmol. 2016; 2016:8421940.
Clinical Ophthalmology

\section{Publish your work in this journal}

Clinical Ophthalmology is an international, peer-reviewed journa covering all subspecialties within ophthalmology. Key topics include: Optometry; Visual science; Pharmacology and drug therapy in eye diseases; Basic Sciences; Primary and Secondary eye care; Patient Safety and Quality of Care Improvements. This journal is indexed on

Submit your manuscript here: http://www.dovepress.com/clinical-ophthalmology-journal

\section{Dovepress}

PubMed Central and CAS, and is the official journal of The Society of Clinical Ophthalmology (SCO). The manuscript management system is completely online and includes a very quick and fair peer-review system, which is all easy to use. Visit http://www.dovepress.com/ testimonials.php to read real quotes from published authors. 